\title{
The Vlasov-Poisson system with radiation damping
}

\author{
Markus Kunze $^{1} \&$ Alan D. Rendall ${ }^{2}$ \\ ${ }^{1}$ Zentrum Mathematik, TU München, \\ Gabelsbergerstr. 49, D- 80333 München, Germany \\ e-mail: mkunze@mathematik.tu-muenchen.de \\ ${ }^{2}$ Max-Planck-Institut für Gravitationsphysik, \\ Am Mühlenberg 1, D- 14476 Golm, Germany \\ e-mail: rendall@aei-potsdam.mpg.de
}

Key words: radiation damping, Vlasov-Poisson system, global existence, long-time behaviour, runaway solutions

\begin{abstract}
We set up and analyze a model of radiation damping within the framework of continuum mechanics, inspired by a model of post-Newtonian hydrodynamics due to Blanchet, Damour and Schäfer. In order to simplify the problem as much as possible we replace the gravitational field by the electromagnetic field and the fluid by kinetic theory. We prove that the resulting system has a well-posed Cauchy problem globally in time for general initial data and that in all solutions the fields decay to zero at late times. In particular, this means that the model is free from the runaway solutions which frequently occur in descriptions of radiation reaction.
\end{abstract}

\section{Introduction and main results}

The Vlasov-Poisson system is a well-known description of collisionless particles which interact via a field which they generate collectively. It can be applied in the case of particles interacting through the electromagnetic field (plasma physics case) or the gravitational field (stellar dynamics case). The equations modelling the two cases are only distinguished by a difference of sign. This description is nonrelativistic and is only appropriate for physical situations where the velocities of the particles are small compared to the velocity of light. When it is replaced by a fully relativistic model the two cases diverge drastically. In the electromagnetic case the appropriate system of equations is the (relativistic) Vlasov-Maxwell system while in the gravitational case it is the Vlasov-Einstein system, which is much more complicated. 
In classical electrodynamics it is well known that accelerated charged particles radiate and that this leads to an effect on the motion of the particles known as radiation reaction. This typically leads to damping, i.e. to loss of energy by the particles. A similar but more complicated effect occurs in the case of the gravitational field. It is, however, hard to formulate exactly due to difficulties such as the nonlinearity and coordinate dependence of the equations used. There is a large literature concerning effective equations in electrodynamics which incorporate radiation damping without providing a full relativistic description of the field and sources. These effective equations usually have undesirable solutions which tend to infinity exponentially fast, the so-called "runaway solutions". It has recently been observed that nevertheless, in some of these models, the physically relevant solutions of the effective equation constitute a center-like manifold in phase space, restricted to which the dynamics is completely well-behaved. Moreover, the effective equation is a good approximation of the full system; cf. [14, 15].

In the case of the gravitational field, radiation damping is a subject of particular interest at the moment due to the fact that gravitational wave detectors will soon be ready to go into operation and it is important for their effective functioning that the sources of gravitational waves be understood well. (For background on gravitational wave detection see for instance [8] and references therein.) The most promising type of source at the moment is a strongly self-gravitating system of two stars rotating about their common centre of mass which lose energy by (gravitational) radiation damping and eventually coalesce. As has already been indicated, it is hard to describe this within the full theory and hence effective equations like those known in electrodynamics are important. Very little is understood about this in terms of rigorous mathematics at this time. The aim of this paper is to take a first step towards bringing this subject into the domain where models can be defined in a mathematically precise way and theorems proved about them.

The model we will discuss has the following characteristics. It clearly exhibits the phenomenon of radiation damping. It does not suffer from pathologies such as runaway solutions. It is simple enough so that we can prove theorems about the global behaviour of the general solution. The particular model was chosen with the aim of obtaining this combination of properties. It is inspired by a model of Blanchet, Damour and Schäfer [5] for a perfect fluid with radiation damping. The phenomenon of radiation damping is intimately connected with the long time asymptotics of the system. Thus, in order to capture it mathematically, we need at least a global existence theorem. This seems hopeless for a fluid, due to the formation of shocks, and so we replace it by collisionless matter (Euler replaced by Vlasov). The latter is known to have good global existence properties [19, 17, 24, 11. Although the original motivation came from the gravitational case, the electromagnetic case is much simpler. Thus we use a model motivated by the electromagnetic case here, hoping to return to the more complicated gravitational case at a later date. We are not aware that the model used here has a direct physical application. 
The model to be studied is defined as follows. There are two species of particles of opposite charges, say ions ("+") and electrons ("-"). In the case of the Vlasov-Poisson system the motion of the individual particles is governed by the characteristic systems

$$
\begin{array}{ll}
\dot{X}^{+}=V^{+}, & \dot{V}^{+}=\nabla U\left(t, X^{+}\right), \\
\dot{X}^{-}=V^{-}, & \dot{V}^{-}=-\nabla U\left(t, X^{-}\right),
\end{array}
$$

where $U=U(t, x)$ is the (electric) potential. The requirement that the particle densities $f^{ \pm}=f^{ \pm}(t, x, v)$ be constant along the characteristics leads to the Vlasov equations

$$
\begin{aligned}
& \partial_{t} f^{+}+v \cdot \nabla_{x} f^{+}+\nabla U \cdot \nabla_{v} f^{+}=0, \\
& \partial_{t} f^{-}+v \cdot \nabla_{x} f^{-}-\nabla U \cdot \nabla_{v} f^{-}=0,
\end{aligned}
$$

with $t \in \mathbb{R}, x \in \mathbb{R}^{3}$, and $v \in \mathbb{R}^{3}$ denoting time, position, and velocity variable, respectively. The potential $U$ derives from the Poisson equation

$$
\Delta U=4 \pi \rho=4 \pi\left(\rho^{+}-\rho^{-}\right), \quad \lim _{|x| \rightarrow \infty} U(t, x)=0,
$$

where

$$
\rho^{ \pm}(t, x)=\int f^{ \pm}(t, x, v) d v .
$$

Supplied with suitable data $f^{ \pm}(t=0)=f_{0}^{ \pm}$, (3)-(6) constitutes the Vlasov-Poisson system for two species of opposite charges; see [9, 22] for general information on Vlasov-Poisson and related models.

In order to introduce a damping effect due to radiation into (3)-(6), we modify the characteristic equations by introducing a small additional term. Let

$$
D(t)=\int x \rho(t, x) d x=\iint x\left(f^{+}(t, x, v)-f^{-}(t, x, v)\right) d x d v
$$

denote the corresponding dipole moment, and replace (11), (2) by

$$
\begin{array}{ll}
\dot{X}^{+}=V^{+}, & \dot{V}^{+}=\nabla U\left(t, X^{+}\right)+\varepsilon \dddot{D}(t), \\
\dot{X}^{-}=V^{-}, & \dot{V}^{-}=-\nabla U\left(t, X^{-}\right)-\varepsilon \dddot{D}(t),
\end{array}
$$

with an $\varepsilon>0$ small. This is to be thought of as an approximation to the full VlasovMaxwell system. It includes the electric dipole radiation which is supposed to give the leading contribution to the radiation reaction, cf. [13, p. 784].

The third time derivative in these equations can lead to pathological behaviour and so we will modify the model by formally small corrections so as to eliminate it. Here we follow the procedure of [5] which was used to tackle the fifth 
time derivatives which occur in the analogous gravitational problem. To reduce the order of derivatives on $D(t)$, we utilize the transformations

$$
\tilde{V}^{+}=V^{+}-\varepsilon \ddot{D}(t) \quad \text { and } \quad \tilde{V}^{-}=V^{-}+\varepsilon \ddot{D}(t) .
$$

Then (8), (9) read

$$
\begin{array}{ll}
\dot{X}^{+}=V^{+}+\varepsilon \ddot{D}(t), & \dot{V}^{+}=\nabla U\left(t, X^{+}\right), \\
\dot{X}^{-}=V^{-}-\varepsilon \ddot{D}(t), & \dot{V}^{-}=-\nabla U\left(t, X^{-}\right),
\end{array}
$$

where the tilde has been omitted for simplicity. The corresponding Vlasov equations are then

$$
\begin{aligned}
& \partial_{t} f^{+}+(v+\varepsilon \ddot{D}(t)) \cdot \nabla_{x} f^{+}+\nabla U \cdot \nabla_{v} f^{+}=0, \\
& \partial_{t} f^{-}+(v-\varepsilon \ddot{D}(t)) \cdot \nabla_{x} f^{-}-\nabla U \cdot \nabla_{v} f^{-}=0 .
\end{aligned}
$$

Next we derive an approximation $D^{[2]}(t)$ to $\ddot{D}(t)$. By definition of $D(t)$ in (7) we formally calculate

$$
\begin{aligned}
\dot{D}(t) & =\iint x\left(\partial_{t} f^{+}-\partial_{t} f^{-}\right) d x d v \\
& =\mathcal{O}(\varepsilon)+\iint x\left(-v \cdot \nabla_{x} f^{+}-\nabla U \cdot \nabla_{v} f^{+}+v \cdot \nabla_{x} f^{-}-\nabla U \cdot \nabla_{v} f^{-}\right) d x d v \\
& =\mathcal{O}(\varepsilon)+\iint v\left(f^{+}-f^{-}\right) d x d v .
\end{aligned}
$$

Thus

$$
\begin{aligned}
\ddot{D}(t) & =\mathcal{O}(\varepsilon)+\iint v\left(\partial_{t} f^{+}-\partial_{t} f^{-}\right) d x d v \\
& =\mathcal{O}(\varepsilon)+\iint v\left(-v \cdot \nabla_{x} f^{+}-\nabla U \cdot \nabla_{v} f^{+}+v \cdot \nabla_{x} f^{-}-\nabla U \cdot \nabla_{v} f^{-}\right) d x d v \\
& =\mathcal{O}(\varepsilon)-\iint v \nabla U \cdot\left(\nabla_{v} f^{+}+\nabla_{v} f^{-}\right) d x d v=\mathcal{O}(\varepsilon)+\iint \nabla U\left(f^{+}+f^{-}\right) d x d v .
\end{aligned}
$$

Hence we are led to define the approximation

$$
\begin{aligned}
D^{[2]}(t) & =\iint \nabla U(t, x)\left(f^{+}(t, x, v)+f^{-}(t, x, v)\right) d x d v \\
& =\int \nabla U(t, x)\left(\rho^{+}(t, x)+\rho^{-}(t, x)\right) d x,
\end{aligned}
$$

and we replace (11), (12) by

$$
\begin{array}{ll}
\dot{X}^{+}=V^{+}+\varepsilon D^{[2]}(t), & \dot{V}^{+}=\nabla U\left(t, X^{+}\right), \\
\dot{X}^{-}=V^{-}-\varepsilon D^{[2]}(t), & \dot{V}^{-}=-\nabla U\left(t, X^{-}\right),
\end{array}
$$


with corresponding Vlasov equations

$$
\begin{aligned}
& \partial_{t} f^{+}+\left(v+\varepsilon D^{[2]}(t)\right) \cdot \nabla_{x} f^{+}+\nabla U \cdot \nabla_{v} f^{+}=0, \\
& \partial_{t} f^{-}+\left(v-\varepsilon D^{[2]}(t)\right) \cdot \nabla_{x} f^{-}-\nabla U \cdot \nabla_{v} f^{-}=0,
\end{aligned}
$$

and $U$ is determined by (5).

We call the system consisting of (18), (19), (5), (6), and (15) the VlasovPoisson system with damping (VPD), and we propose it as a model to study the damping effect due to radiation. We add some more comments.

Remark 1 (a) To model radiation as we have done it, it is necessary to consider at least two species with different charge to mass ratios. Here we make the simplest choice of equal masses and two charges which are equal in magnitude and opposite in sign. If the charge to mass ratios were equal then the rate of change of the dipole moment would be proportional to the linear momentum of the system and, by conservation of momentum, the radiation reaction force would vanish. This is a well-known fact (absence of bremsstrahlung for identical particles), cf. [7, p. 411] or [25, p. 201], and can also be seen from the corresponding effective equations for radiation reaction, cf. [16, eq. after (1.9)].

(b) In the context of general relativity, one has to use the quadrupole moment

$$
Q_{i j}(t)=\int\left(x_{i} x_{j}-\frac{1}{3}|x|^{2} \delta_{i j}\right) \rho(t, x) d x
$$

instead of the dipole moment $D(t)$ and it is the fifth time derivative which occurs instead of the third before reduction [7]. This leads to considerable complications.

(c) Notice that $D^{[2]}(t) \equiv 0$ for e.g. spherically symmetric solutions, whence there is no radiation damping in this case.

It is the purpose of this paper to analyze rigorously long-time properties of classical solutions to (VPD). Therefore we first have to deal with the question of global existence of solutions, e.g. for smooth data functions $f^{ \pm}(t=0)=f_{0}^{ \pm}$of compact support, i.e. such that

$$
f_{0}^{ \pm} \in C_{0}^{\infty}\left(\mathbb{R}^{3} \times \mathbb{R}^{3}\right), \quad f_{0}^{ \pm} \geq 0, \quad \text { and } \quad f_{0}^{ \pm}(x, v)=0 \quad \text { for } \quad|x| \geq r_{0} \text { or }|v| \geq r_{0}
$$

with some fixed $r_{0}>0$. Since global existence is a quite non-trivial issue for VlasovPoisson like systems, we provide a complete existence proof for (VPD) in section 3, deriving estimates on higher velocity moments of $f^{ \pm}$along the lines of 17. This approach has been successfully applied to other related problems as well; cf. [2, 6].

In this manner we obtain

Theorem 1 If $f_{0}^{ \pm}$satisfy (20), then there is a unique solution

$$
f^{ \pm} \in C^{1}\left(\left[0, \infty\left[\times \mathbb{R}^{3} \times \mathbb{R}^{3}\right)\right.\right.
$$

of (VPD) with data $f^{ \pm}(t=0)=f_{0}^{ \pm}$. 
Having ensured that suitable solutions do exist, we now turn to the decay estimates for quantities related to (VPD). We define the total energy

$$
\mathcal{E}(t)=\mathcal{E}_{\text {kin }}(t)+\mathcal{E}_{\text {pot }}(t)
$$

with

$$
\begin{aligned}
& \mathcal{E}_{\text {kin }}(t)=\frac{1}{2} \iint|v|^{2}\left(f^{+}(t, x, v)+f^{-}(t, x, v)\right) d x d v, \quad \text { and } \\
& \mathcal{E}_{\text {pot }}(t)=\frac{1}{8 \pi} \int|\nabla U(t, x)|^{2} d x
\end{aligned}
$$

denoting kinetic and potential energy, respectively.

Theorem 2 Assume $f_{0}^{ \pm}$satisfy (20). Then

$$
\dot{\mathcal{E}}(t)=-\varepsilon\left|D^{[2]}(t)\right|^{2}
$$

Moreover, the following estimates hold for $t \in[0, \infty[$.

(a) $\left\|\rho^{ \pm}(t)\right\|_{p ; x} \leq C(1+t)^{-\frac{3(p-1)}{2 p}}$ for $p \in\left[1, \frac{5}{3}\right]$;

(b) $\|\nabla U(t)\|_{p ; x} \leq C(1+t)^{-\frac{5 p-3}{7 p}}$ for $p \in\left[2, \frac{15}{4}\right]$;

(c) $\left|D^{[2]}(t)\right| \leq C(1+t)^{-\frac{8}{7}}$.

In particular, (VPD) does not admit nontrivial static solutions, and the kinetic energy satisfies $\mathcal{E}_{\mathrm{kin}}(t) \rightarrow \mathcal{E}_{\infty}$ as $t \rightarrow \infty$ for some $\mathcal{E}_{\infty} \geq 0$. Moreover, if $\mathcal{E}(0)>0$ and $\varepsilon>0$ is small enough, then $\mathcal{E}_{\infty}>0$.

See Section 2 for the proof. We note that in theorem 2 a slow dissipation of energy takes place due to the "damping term" $D^{[2]}(t)$, as can be seen from equation $(23)$.

Remark 2 As an aside, we include a comment on a relation to the usual VlasovPoisson system. We start with the characteristic equations (16), (17), i.e.

$$
\begin{array}{ll}
\dot{X}^{+}=V^{+}+\varepsilon D^{[2]}(t), & \dot{V}^{+}=\nabla U\left(t, X^{+}\right), \\
\dot{X}^{-}=V^{-}-\varepsilon D^{[2]}(t), & \dot{V}^{-}=-\nabla U\left(t, X^{-}\right) .
\end{array}
$$

Define

$$
\begin{array}{ll}
\bar{X}^{+}=X^{+}, & \bar{V}^{+}=V^{+}+\varepsilon D^{[2]}(t), \\
\bar{X}^{-}=X^{-}, & \bar{V}^{-}=V^{-}-\varepsilon D^{[2]}(t) .
\end{array}
$$


Then

$$
\begin{aligned}
& \dot{\bar{X}}^{+}=\bar{V}^{+}, \quad \dot{\bar{V}}^{+}=\nabla U\left(t, \bar{X}^{+}\right)+\varepsilon \dot{D}^{[2]}(t)=\nabla W\left(t, \bar{X}^{+}\right), \\
& \dot{\bar{X}}^{-}=\bar{V}^{-}, \quad \dot{\bar{V}}^{-}=-\nabla U\left(t, \bar{X}^{-}\right)-\varepsilon \dot{D}^{[2]}(t)=-\nabla W\left(t, \bar{X}^{-}\right),
\end{aligned}
$$

where

$$
W(t, x)=U(t, x)+\varepsilon \dot{D}^{[2]}(t) \cdot x .
$$

Also $\Delta W=\Delta U$. Thus we obtain a solution of the Vlasov-Poisson system where the potential $W$ does not satisfy the usual boundary conditions. This is similar to the cosmological solutions of the Vlasov-Poisson system constructed in [23. They are obtained directly as solutions of a transformed system but are in the end solutions of the Vlasov-Poisson system with unconventional boundary conditions. This reformulation gives a simple way of seeing the volume preserving property of the flow for (VPD), since we know it for Vlasov-Poisson. It is, however, not hard to see it directly.

Notation. Throughout the paper, $C$ denotes a general constant which may change from line to line and which only depends on $f_{0}^{ \pm}$. If we consider a solution on a fixed time interval $[0, T]$, and if $C$ additionally depends on $T$, this is indicated by $C_{T}$. The usual $L^{p}$-norm of a function $\varphi=\varphi(t, x)$ over $x \in \mathbb{R}^{3}$ is denoted by $\|\varphi(t)\|_{p ; x}$, and if $\varphi=\varphi(t, x, v)$ and the integrals are to be extended over $(x, v) \in \mathbb{R}^{3} \times \mathbb{R}^{3}$, then we write $\|\varphi(t)\|_{p ; x v}$. To simplify notation, an integral $\int$ always means $\int_{\mathbb{R}^{3}}$.

Acknowledgements. We wish to thank Thibault Damour, Gerhard Rein, Gerhard Schäfer and Herbert Spohn for discussions and helpful advice. MK acknowledges support through a Heisenberg fellowship of DFG.

\section{Proof of theorem 2}

We split the proof into several subsections.

\subsection{Energy dissipation}

We verify (23) and calculate the change of the total energy $\mathcal{E}(t)$ from (21). Due to (18) and (19) we have

$$
\begin{aligned}
\dot{\mathcal{E}}_{\text {kin }}(t)= & \frac{1}{2} \iint v^{2}\left(\partial_{t} f^{+}+\partial_{t} f^{-}\right) d x d v \\
= & \frac{1}{2} \iint v^{2}\left(-\left[v+\varepsilon D^{[2]}(t)\right] \cdot \nabla_{x} f^{+}-\nabla U \cdot \nabla_{v} f^{+}\right. \\
& \left.\quad-\left[v-\varepsilon D^{[2]}(t)\right] \cdot \nabla_{x} f^{-}+\nabla U \cdot \nabla_{v} f^{-}\right) d x d v \\
= & \iint(v \cdot \nabla U)\left(f^{+}-f^{-}\right) d x d v=\int \nabla U \cdot j d x,
\end{aligned}
$$


where

$$
j(t, x)=j^{+}(t, x)-j^{-}(t, x), \quad j^{ \pm}(t, x)=\int v f^{ \pm}(t, x, v) d v,
$$

is the current. The evaluation of $\dot{\mathcal{E}}_{\text {pot }}(t)$ is a little more tedious, and for this purpose we will use

$$
\mathcal{E}_{\mathrm{pot}}(t)=\frac{1}{8 \pi} \int d x|\nabla U|^{2}=-\frac{1}{8 \pi} \int d x(\Delta U) U=\frac{1}{2} \int d x\left(\rho^{-}-\rho^{+}\right) U,
$$

and moreover the representations of the electric field by means of Coulomb potentials

$$
\begin{aligned}
U(t, x) & =-\int \frac{d y}{|x-y|}\left(\rho^{+}(t, y)-\rho^{-}(t, y)\right), \\
E(t, x) & :=\nabla U(t, x)=-\int d y \nabla_{x} \frac{1}{|x-y|}\left(\rho^{+}(t, y)-\rho^{-}(t, y)\right) \\
& =\int d y \frac{(x-y)}{|x-y|^{3}}\left(\rho^{+}(t, y)-\rho^{-}(t, y)\right) .
\end{aligned}
$$

Then we obtain through the change of variables $x \leftrightarrow y$ and $v \leftrightarrow w$

$$
\begin{gathered}
\dot{\mathcal{E}}_{\text {pot }}(t) \\
=\frac{1}{2} \iint d x d v\left\{\left(\partial_{t} \rho^{-}-\partial_{t} \rho^{+}\right) U+\left(\rho^{-}-\rho^{+}\right)\left(\partial_{t} U\right)\right\} \\
=-\frac{1}{2} \iiint \int d x d y d v d w\left\{\left(\partial_{t} f^{-}(t, x, v)-\partial_{t} f^{+}(t, x, v)\right)\right. \\
\times \frac{1}{|x-y|}\left(f^{+}(t, y, w)-f^{-}(t, y, w)\right) \\
+\left(f^{-}(t, x, v)-f^{+}(t, x, v)\right) \\
\left.\times \frac{1}{|x-y|}\left(\partial_{t} f^{+}(t, y, w)-\partial_{t} f^{-}(t, y, w)\right)\right\} \\
=-\iiint \int d x d y d v d w\left(\partial_{t} f^{-}(t, x, v)-\partial_{t} f^{+}(t, x, v)\right) \\
\times \frac{1}{|x-y|}\left(f^{+}(t, y, w)-f^{-}(t, y, w)\right) \\
=\iiint \int \frac{d x d y}{|x-y|} d v d w\left\{\left[v-\varepsilon D^{[2]}(t)\right] \cdot \nabla_{x} f^{-}(t, x, v)-\nabla U(t, x) \cdot \nabla_{v} f^{-}(t, x, v)\right. \\
\left.-\left[v+\varepsilon D^{[2]}(t)\right] \cdot \nabla_{x} f^{+}(t, x, v)-\nabla U(t, x) \cdot \nabla_{v} f^{+}(t, x, v)\right\} \\
\times\left(f^{+}(t, y, w)-f^{-}(t, y, w)\right) \\
=\iiint \frac{d x d y}{|x-y|} d v\left\{\left[v-\varepsilon D^{[2]}(t)\right] \cdot \nabla_{x} f^{-}(t, x, v)-\left[v+\varepsilon D^{[2]}(t)\right] \cdot \nabla_{x} f^{+}(t, x, v)\right\}
\end{gathered}
$$




$$
\begin{aligned}
& \times\left(\rho^{+}(t, y)-\rho^{-}(t, y)\right) \\
&=-\iiint d x d y d v\left(\nabla_{x} \frac{1}{|x-y|}\right) \cdot\left\{\left[v-\varepsilon D^{[2]}(t)\right] f^{-}(t, x, v)\right. \\
&\left.-\left[v+\varepsilon D^{[2]}(t)\right] f^{+}(t, x, v)\right\}\left(\rho^{+}(t, y)-\rho^{-}(t, y)\right)
\end{aligned}
$$

recall the definition of $D^{[2]}(t)$ from (15). Combining this with (24), we see that (23) holds.

\subsection{Decay of the potential energy}

Here we show a $t^{-1}$-decay of the potential energy $\mathcal{E}_{\text {pot }}(t)$ from (25). The result is similar to [12, 18], but the proof requires appropriate modifications due to the presence of the term $D^{[2]}(t)$.

Lemma 1 We have

$$
\mathcal{E}_{\text {pot }}(t) \leq C(1+t)^{-1} \quad \text { and } \quad \iint(x-v t)^{2}\left(f^{+}+f^{-}\right) d x d v \leq C t, \quad t \in[0, \infty[,
$$

the constants being independent of $\varepsilon \in[0,1]$.

Proof : Denote

$$
R(t)=\iint(x-v t)^{2}\left(f^{+}+f^{-}\right) d x d v \quad \text { and } \quad g(t)=\frac{t^{2}}{4 \pi} \int|\nabla U|^{2} d x=2 t^{2} \mathcal{E}_{\mathrm{pot}}(t) .
$$

Then a short calculation reveals

$$
\begin{aligned}
\dot{R}(t)= & -2 t \int(x \cdot \nabla U) \rho d x+2 t^{2} \int \nabla U \cdot j d x \\
& +2 \varepsilon D^{[2]}(t) \cdot\left(\iint(x-t v)\left(f^{+}-f^{-}\right) d x d v\right) .
\end{aligned}
$$

Inserting 26] for $\nabla U$ and with $x \cdot(x-y)|x-y|^{-3}=|x-y|^{-1}+y \cdot(x-y)|x-y|^{-3}$, we see that

$$
\int(x \cdot \nabla U) \rho d x=-\frac{1}{2} \int U \rho d x=\frac{1}{8 \pi} \int|\nabla U|^{2} d x .
$$


On the other hand, (24) and the energy identity (23) imply

$$
\int \nabla U \cdot j d x=\dot{\mathcal{E}}_{\text {kin }}(t)=-\dot{\mathcal{E}}_{\text {pot }}(t)-\varepsilon\left|D^{[2]}(t)\right|^{2}=-\frac{1}{8 \pi} \frac{d}{d t} \int|\nabla U|^{2} d x-\varepsilon\left|D^{[2]}(t)\right|^{2} .
$$

Using (29) and (30) in (28), it follows that

$$
\begin{aligned}
\dot{R}(t)= & -\frac{t}{4 \pi} \int|\nabla U|^{2} d x+2 t^{2}\left(-\frac{1}{8 \pi} \frac{d}{d t} \int|\nabla U|^{2} d x-\varepsilon\left|D^{[2]}(t)\right|^{2}\right) \\
& +2 \varepsilon D^{[2]}(t) \cdot\left(\iint(x-t v)\left(f^{+}-f^{-}\right) d x d v\right) .
\end{aligned}
$$

By means of $g$ from (27), this may be rewritten as

$$
\begin{aligned}
\frac{d}{d t}(R(t)+g(t))= & \frac{g(t)}{t}-2 \varepsilon t^{2}\left|D^{[2]}(t)\right|^{2} \\
& +2 \varepsilon D^{[2]}(t) \cdot\left(\iint(x-t v)\left(f^{+}-f^{-}\right) d x d v\right) .
\end{aligned}
$$

Compared to [12, p. 1412], it is now necessary to see how the two terms with $D^{[2]}(t)$ contribute.

First we consider the case that $t>0$ is such that

$$
t^{2}\left|D^{[2]}(t)\right| \leq\left|\iint(x-t v)\left(f^{+}-f^{-}\right) d x d v\right| .
$$

Then we obtain from (31) that

$$
\begin{aligned}
\frac{d}{d t}(R(t)+g(t)) & \leq \frac{g(t)}{t}+2 \varepsilon\left|D^{[2]}(t)\right|\left|\iint(x-t v)\left(f^{+}-f^{-}\right) d x d v\right| \\
& \leq \frac{g(t)}{t}+2 \varepsilon t^{-2}\left|\iint(x-t v)\left(f^{+}-f^{-}\right) d x d v\right|^{2}
\end{aligned}
$$

However, if

$$
t^{2}\left|D^{[2]}(t)\right| \geq\left|\iint(x-t v)\left(f^{+}-f^{-}\right) d x d v\right|
$$

then (31) yields

$$
\frac{d}{d t}(R(t)+g(t)) \leq \frac{g(t)}{t},
$$

hence (32) is verified for all $t>0$. In order to have bounds below independent of, say, $\varepsilon \in[0,1]$, we modify (32) to

$$
\frac{d}{d t}(R(t)+g(t)) \leq \frac{g(t)}{t}+2 t^{-2}\left|\iint(x-t v)\left(f^{+}-f^{-}\right) d x d v\right|^{2}
$$


To further exploit this, we next note that due to Hölder's inequality and by lemma 2 below with $p=0$

$$
\begin{aligned}
& \left|\iint(x-t v)\left(f^{+}-f^{-}\right) d x d v\right|^{2} \\
& \leq\left(\iint(x-t v)^{2}\left[f^{+}+f^{-}\right] d x d v\right)\left(\iint\left[f^{+}+f^{-}\right] d x d v\right) \leq C R(t),
\end{aligned}
$$

thus by (33)

$$
\frac{d}{d t}(R(t)+g(t)) \leq \frac{g(t)}{t}+C t^{-2} R(t), \quad t>0 .
$$

Integrating over $t \in[1, T]$, we see that

$$
R(T) \leq R(T)+g(T) \leq C+\int_{1}^{T} \frac{g(t)}{t} d t+C \int_{1}^{T} t^{-2} R(t) d t, \quad T \geq 1 .
$$

Therefore

$$
R(T) \leq C\left(1+\int_{1}^{T} \frac{g(t)}{t} d t\right), \quad T \geq 1
$$

by Gronwall's lemma. Using this in (35), we find

$$
\begin{aligned}
g(T) & \leq C+\int_{1}^{T} \frac{g(t)}{t} d t+C \int_{1}^{T}\left(1+\int_{1}^{t} \frac{g(s)}{s} d s\right) \frac{d t}{t^{2}} \\
& \leq C+\int_{1}^{T} \frac{g(t)}{t} d t+C \int_{1}^{T}\left(\frac{1}{t}-\frac{1}{T}\right) \frac{g(t)}{t} d t,
\end{aligned}
$$

and consequently

$$
g(T) \leq C T, \quad T \geq 1,
$$

again by Gronwall's lemma. According to the definition of $g$, this proves the $t^{-1}$ decay of $\mathcal{E}_{\text {pot }}(t)$, and then (36) shows that $R(T) \leq C T$ holds as well. This completes the proof of lemma 1 .

\subsection{Some general estimates}

We digress now from the proof of theorem 2 and note some useful estimates that will also play a role later for the global existence of solutions, cf. theorem 1. For $p \in[0, \infty[$ define the velocity moments

$$
M_{p}^{ \pm}(t)=\iint|v|^{p} f^{ \pm}(t, x, v) d x d v, \quad \text { and } \quad M_{p}(t)=\sup _{s \in[0, t]}\left(M_{p}^{+}(s)+M_{p}^{-}(s)\right) .
$$


Lemma 2 For $t \in[0, \infty[$ we have

$$
\left\|f^{ \pm}(t)\right\|_{\infty ; x v} \leq C \quad \text { and } \quad M_{p}(t) \leq C, \quad p \in[0,2] .
$$

Proof: For fixed $t \in[0, \infty[$ let $(\mathcal{X}(s), \mathcal{V}(s))=(\mathcal{X}(s ; t, x, v), \mathcal{V}(s ; t, x, v))$ denote the characteristics from (16) associated with (18), i.e.

$$
\left(\begin{array}{c}
\dot{\mathcal{X}}(s) \\
\dot{\mathcal{V}}(s)
\end{array}\right)=\left(\begin{array}{c}
\mathcal{V}(s)+\varepsilon D^{[2]}(s) \\
\nabla U(s, \mathcal{X}(s))
\end{array}\right), \quad\left(\begin{array}{c}
\mathcal{X}(t) \\
\mathcal{V}(t)
\end{array}\right)=\left(\begin{array}{c}
x \\
v
\end{array}\right)
$$

Then $\frac{\partial}{\partial s}\left[f^{+}(s, \mathcal{X}(s), \mathcal{V}(s))\right]=0$ shows $f^{+}(t, x, v)=f_{0}^{+}(\mathcal{X}(0), \mathcal{V}(0))$, and hence the first bound follows. Concerning the second,

$$
M_{2}(t)=2 \sup _{s \in[0, t]} \mathcal{E}_{\text {kin }}(s) \leq 2 \sup _{s \in[0, t]} \mathcal{E}(s)=2 \mathcal{E}(0)
$$

by (23). Also $M_{0}^{ \pm}(t)=M_{0}^{ \pm}(0)$, as $(x, v) \mapsto(\mathcal{X}(0 ; t, x, v), \mathcal{V}(0 ; t, x, v))$ is a volumepreserving diffeomorphism of $\mathbb{R}^{3} \times \mathbb{R}^{3}$, due to the fact that the right-hand side of the ODE in (38) has divergence $\operatorname{div}=\operatorname{div}_{(\mathcal{X}, \mathcal{V})}$ zero; see also lemma 16 below and remark 2. Observing that $|v|^{p} \leq 1+|v|^{2}$ for $v \in \mathbb{R}^{3}$ and $p \in[0,2]$ completes the proof.

Lemma 3 Let $f=f(x, v) \in L^{\infty}\left(\mathbb{R}^{3} \times \mathbb{R}^{3}\right)$ be a nonnegative function such that $\iint|v|^{p} f(x, v) d x d v<\infty$ for some $p \in\left[0, \infty\left[\right.\right.$, and define $\phi(x)=\int f(x, v) d v, x \in \mathbb{R}^{3}$. Then

$$
\|\phi\|_{\frac{3+p}{3} ; x} \leq C\|f\|_{\infty ; x v}^{\frac{p}{3+p}}\left(\iint|v|^{p} f(x, v) d x d v\right)^{\frac{3}{3+p}} .
$$

Here $C$ depends only on $p$.

Proof : The argument is well-known, but indicated for completeness. We split

$$
\phi(x) \leq \int_{|v| \leq R} f(x, v) d v+\int_{|v| \geq R} f(x, v) d v \leq \frac{4 \pi}{3} R^{3}\|f\|_{\infty ; x v}+R^{-p} \int|v|^{p} f(x, v) d v
$$

and optimize in $R$ to find

$$
\phi(x) \leq C\|f\|_{\infty ; x v}^{\frac{p}{3+p}}\left(\int|v|^{p} f(x, v) d v\right)^{\frac{3}{3+p}},
$$

whence integration w.r.t. $x$ yields (39). 
Lemma 4 We have

$$
\left.\left\|\left(\nabla \frac{1}{|x|}\right) * \rho\right\|_{q ; x} \leq C\|\rho\|_{p ; x}, \quad q \in\right] \frac{3}{2}, \infty\left[, \quad p=\frac{3 q}{3+q} .\right.
$$

In addition,

$$
\left.\left\|\left(\nabla \frac{1}{|x|}\right) * \operatorname{div} \Gamma\right\|_{q ; x} \leq C\|\Gamma\|_{q ; x}, \quad q \in\right] 1, \infty[,
$$

for smooth and compactly supported vector fields $\Gamma: \mathbb{R}^{3} \rightarrow \mathbb{R}^{3}$.

Proof: The first estimate is a consequence of the classical Hardy-LittlewoodSobolev inequality; see [10, Thm. 4.5.3]. Concerning the second, we note that integration by parts reveals

$$
\int \frac{x-y}{|x-y|^{3}} \operatorname{div} \Gamma(y) d y=\frac{4 \pi}{3} \Gamma(x)-\lim _{\varepsilon \rightarrow 0} \int_{|x-y| \geq \varepsilon} \Gamma(x-y) \cdot g(y) d y,
$$

with $g(y)=\frac{1}{|y|^{3}} G(y)$, where $G(y)=(-\mathrm{Id})+\frac{3}{|y|^{2}}(y \otimes y) \in \mathbb{R}^{3 \times 3}$. Since $G$ is bounded in $\mathbb{R}^{3} \backslash\{0\}$, homogeneous of degree zero, and satisfies $\int_{|y|=1} G(y) d^{2} y=0$, the Calderón-Zygmund inequality [1, Thm. 4.31] implies that the second term on the right-hand side of (40) defines a bounded operator $L^{q}\left(\mathbb{R}^{3}\right) \rightarrow L^{q}\left(\mathbb{R}^{3}\right)$; in view of the compact support of the $\Gamma^{\prime}$ 's it is not necessary that $G$ has compact support.

Lemma 5 For $t \in[0, \infty[$ we have

$$
\left\|\rho^{ \pm}(t)\right\|_{p ; x} \leq C M_{3(p-1)}(t)^{\frac{1}{p}}, \quad p \in[1, \infty[,
$$

as well as

$$
\left.\|\nabla U(t)\|_{q ; x} \leq C\|\rho(t)\|_{\frac{3 q}{3+q} ; x} \leq C M_{\frac{6 q-9}{3+q}}(t)^{\frac{3+q}{3 q}}, \quad q \in\right] \frac{3}{2}, \infty[.
$$

Moreover,

$$
\left|D^{[2]}(t)\right| \leq\|\nabla U(t)\|_{p ; x}\|\rho(t)\|_{p^{\prime} ; x}, \quad p \in[1, \infty] .
$$

Proof : According to lemma 3 and lemma 2 ,

$$
\left\|\rho^{ \pm}(t)\right\|_{\frac{3+\alpha}{3} ; x} \leq C\left\|f^{ \pm}(t)\right\|_{\infty ; x v}^{\frac{\alpha}{3+\alpha}} M_{\alpha}(t)^{\frac{3}{3+\alpha}} \leq C M_{\alpha}(t)^{\frac{3}{3+\alpha}}
$$

for all $\alpha \geq 0$, hence (41) holds. Due to (26) we see lemma 1 applies to yield, for $q \in] \frac{3}{2}, \infty\left[\right.$ and with $p=\frac{3 q}{3+q}$, together with (41)

$$
\|\nabla U(t)\|_{q ; x} \leq C\left(\left\|\rho^{+}(t)\right\|_{p ; x}+\left\|\rho^{-}(t)\right\|_{p ; x}\right) \leq C M_{3(p-1)}(t)^{\frac{1}{p}} .
$$

Expressing $p$ through $q$, we arrive at (42). The estimate on $\left|D^{[2]}(t)\right|$ is a consequence of (15) and Hölder's inequality. 


\subsection{Proof of theorem 2 (completed)}

From lemma 1 we additionally obtain, analogously to [12], the following information.

Corollary 1 Under the assumptions of theorem 园, we moreover have

$$
\left\|\rho^{ \pm}(t)\right\|_{\frac{5}{3} ; x} \leq C(1+t)^{-3 / 5}, \quad t \in[0, \infty[,
$$

and

$$
\|\nabla U(t)\|_{\frac{15}{4} ; x} \leq C(1+t)^{-3 / 5}, \quad t \in[0, \infty[.
$$

Proof: Using lemma 2 and lemma 1 we can split

$$
\begin{aligned}
\rho^{ \pm}(t, x) & \leq \int_{\{v:|x-t v| \leq R\}} f^{ \pm}(t, x, v) d v+R^{-2} \int_{\{v:|x-t v| \geq R\}}(x-t v)^{2} f^{ \pm}(t, x, v) d v \\
& \leq C R^{3} t^{-3}+R^{-2} \int(x-t v)^{2}\left(f^{+}+f^{-}\right)(t, x, v) d v \leq C R^{3} t^{-3}+C R^{-2} t,
\end{aligned}
$$

and then choose the optimal $R \cong t^{4 / 5}$ to obtain (44). Concerning (45), this follows from (44) and the first inequality in (42) with $q=\frac{15}{4}$.

Lemma 6 Assertions (a)-(c) of theorem 圆 are satisfied.

Proof : From lemma 2 and corollary 1 we know that $\left\|\rho^{ \pm}(t)\right\|_{1 ; x} \leq C$, and moreover $\left\|\rho^{ \pm}(t)\right\|_{\frac{5}{3} ; x} \leq C(1+t)^{-3 / 5}$. We may also estimate $\|\nabla U(t)\|_{2 ; x}=\sqrt{8 \pi} \mathcal{E}_{\text {pot }}(t)^{1 / 2} \leq$ $C(1+t)^{-1 / 2}$ as well as $\|\nabla U(t)\|_{\frac{15}{4} ; x} \leq C(1+t)^{-3 / 5}$ by lemma 1 and corollary 1. Hence the general interpolation estimate

$$
\|\phi\|_{p} \leq\|\phi\|_{q_{1}}^{\alpha}\|\phi\|_{q_{2}}^{1-\alpha}, \quad p \in\left[q_{1}, q_{2}\right], \quad \frac{1}{p}=\frac{\alpha}{q_{1}}+\frac{1-\alpha}{q_{2}},
$$

yields (a) and (b). For (c), we use (43) with $p=5 / 2$ and $p^{\prime}=5 / 3$, (a), and (b) to see that

$$
\left|D^{[2]}(t)\right| \leq\|\nabla U(t)\|_{\frac{5}{2} ; x}\|\rho(t)\|_{\frac{5}{3} ; x} \leq C(1+t)^{-19 / 35}(1+t)^{-3 / 5}=C(1+t)^{-8 / 7},
$$

as was to be shown.

Remark 3 The estimates derived thus far suggest that the optimal decay rate for $D^{[2]}(t)$ be $\left|D^{[2]}(t)\right| \sim t^{-3 / 2}$ rather than $\left|D^{[2]}(t)\right| \sim t^{-8 / 7}$, for the following reason: by Hölder's inequality and lemma 11 the quantity $I(t)=\iint(x-v t)\left(f^{+}-f^{-}\right) d x d v$ satisfies

$$
|I(t)| \leq C\left(\iint(x-v t)^{2}\left(f^{+}+f^{-}\right) d x d v\right)^{1 / 2} \leq C(1+t)^{1 / 2},
$$


thus we might expect $\dot{I}(t) \sim t^{-1 / 2}$. On the other hand, direct calculation shows

$$
\dot{I}(t)=\left(\varepsilon \iint\left(f^{+}+f^{-}\right) d x d v-t\right) D^{[2]}(t) \sim(-t) D^{[2]}(t),
$$

whence we should have $\left|D^{[2]}(t)\right| \sim t^{-3 / 2}$. This decay would also be obtained if it were possible to use theorem 2 (a) and (b) with $p=\frac{15}{4}$ and $p=\frac{15}{11}$, respectively, since then (43) would yield

$$
\begin{aligned}
\left|D^{[2]}(t)\right| & \leq\|\nabla U(t)\|_{\frac{15}{11} ; x}\|\rho(t)\|_{\frac{15}{4} ; x} \\
& \leq C(1+t)^{-\frac{5(15 / 11)-3}{7(15 / 11)}}(1+t)^{-\frac{3((15 / 4)-1)}{2(15 / 4)}}=C(1+t)^{-3 / 2} .
\end{aligned}
$$

However, the necessary decay estimates for such $p$-norms of $\nabla U(t)$ and $\rho(t)$ could not be proved.

Corollary 2 There are no nontrivial static solutions of (VPD), and $\mathcal{E}_{\text {kin }}(t) \rightarrow$ $\mathcal{E}_{\infty} \geq 0$ as $t \rightarrow \infty$. If $\mathcal{E}(0)>0$ and $\varepsilon>0$ is sufficiently small, then $\mathcal{E}_{\infty}>0$.

Proof : If (VPD) had a static solution $f^{ \pm}(t) \equiv f_{0}^{ \pm}$, then $\mathcal{E}_{\text {pot }}(t) \equiv 0$, whence $\nabla U=$ 0 . This in turn yields $D^{[2]}(t) \equiv 0$ by definition. Consequently, the Vlasov equations (18), (19) reduce to $\partial_{t} f^{ \pm}+v \cdot \nabla_{x} f^{ \pm}=0$ with unique solution $f^{ \pm}(x, v)=f_{0}^{ \pm}(x-$ $v t, v)$. But then we see $\rho^{ \pm}(x)=\int f_{0}^{ \pm}(x-v t, v) d v=t^{-3} \int f_{0}^{ \pm}\left(w, t^{-1}[x-w]\right) d w$, showing as $t \rightarrow \infty$ that the solution has to be trivial. To prove the assertion concerning $\mathcal{E}_{\text {kin }}(t)$, note that, since $\mathcal{E}(t)$ is decaying by (23), $\mathcal{E}(t) \rightarrow \mathcal{E}_{\infty} \geq 0$ as $t \rightarrow \infty$. But $\mathcal{E}(t)=\mathcal{E}_{\text {kin }}(t)+\mathcal{E}_{\text {pot }}(t)$ and $\mathcal{E}_{\text {pot }}(t) \rightarrow 0$, hence the first claim follows. For the second, denote $C_{1}$ the constant on the right-hand side of (46). Since all bounds are derived from lemma $\mathbb{1}$, we note that $C_{1}$ is independent of $\varepsilon \in[0,1]$. Integrating (23) yields

$$
\mathcal{E}_{\text {kin }}(t)+\mathcal{E}_{\text {pot }}(t)=\mathcal{E}(0)-\varepsilon \int_{0}^{t}\left|D^{[2]}(s)\right|^{2} d s,
$$

thus as $t \rightarrow \infty$, provided that $\mathcal{E}_{\infty}=0$, by 46

$$
\mathcal{E}(0)=\varepsilon \int_{0}^{\infty}\left|D^{[2]}(s)\right|^{2} d s \leq C_{1}^{2} \varepsilon \int_{0}^{\infty}(1+s)^{-16 / 7} d s=\left(7 C_{1}^{2} / 9\right) \varepsilon
$$

So if we choose $\varepsilon<\left(9 / 7 C_{1}^{2}\right) \mathcal{E}(0)$, then necessarily $\mathcal{E}_{\infty}>0$.

Taking into account Section 2.1, lemma 6, and corollary 2, we note that the proof of theorem 2 is complete.

Remark 4 With regard to corollary 2, $\mathcal{E}_{\infty}=\lim _{t \rightarrow \infty} \mathcal{E}_{\text {kin }}(t)>0$ was to be expected, since otherwise the particle velocities would have to tend to zero. It is, however, not surprising that at late times, when we are in a small data regime and the radiation reaction force is getting small, the solution behaves like a solution of the Vlasov-Poisson system with small data. In that case the particles travel with constant non-zero velocity at late times, as shown in [3]. 
We note a further consequence of the foregoing estimates.

Corollary 3 If $\mathcal{E}_{\infty}>0$, then

$$
C_{1} t-C_{2} \leq \iint(x \cdot v)\left(f^{+}(t, x, v)+f^{-}(t, x, v)\right) d x d v \leq C_{3}(1+t), \quad t \in[0, \infty[
$$

for constants $C_{1}, C_{2}, C_{3}>0$.

Proof: Denote $S(t)=\iint(x \cdot v)\left(f^{+}+f^{-}\right) d x d v$. In view of lemma 11 we obtain

$$
\begin{aligned}
2 t^{2} \mathcal{E}_{\mathrm{kin}}(t) & =\iint(x-v t)^{2}\left(f^{+}+f^{-}\right) d x d v-\iint x^{2}\left(f^{+}+f^{-}\right) d x d v+2 t S(t) \\
& \leq C(1+t)+2 t S(t)
\end{aligned}
$$

whence $t^{2} \mathcal{E}_{\infty} \leq C(1+t)+2 t S(t)$ for $t$ large enough. To prove the upper bound, note that $Q(t)=\iint x^{2}\left(f^{+}+f^{-}\right) d x d v$ satisfies $\dot{Q}(t)=2 S(t)+2 \varepsilon D^{[2]}(t) \cdot \iint x\left(f^{+}-\right.$ $\left.f^{-}\right) d x d v$, as follows by a straightforward calculation. Therefore utilizing Hölder's inequality we obtain

$$
\dot{Q}(t) \leq 2 Q(t)^{1 / 2}\left(2 \mathcal{E}_{\text {kin }}(t)\right)^{1 / 2}+C(1+t)^{-8 / 7} Q(t)^{1 / 2} \leq C Q(t)^{1 / 2} .
$$

Consequently, $Q(t) \leq C(1+t)^{2}$, and this in turn yields, once more by Hölder's inequality, $|S(t)| \leq Q(t)^{1 / 2}\left(2 \mathcal{E}_{\text {kin }}(t)\right)^{1 / 2} \leq C(1+t)$.

\section{Existence of solutions}

As mentioned in the introduction, the proof follows [17. The idea is to decompose the field $E=\nabla U=E_{1}+F$ in a "far field" $F$ that is small, and in some complementary part $E_{1}$ which is of higher regularity than $E$ itself. (More precisely, $\left\|E_{1}(t)\right\|_{p ; x} \leq C$ for every $p \in\left[1, \frac{15}{4}\right.$ (can be achieved.) According to this splitting, we write the Vlasov equations (18) and (19) in the form

$$
\begin{aligned}
& \partial_{t} f^{+}+\left(v+\varepsilon D^{[2]}(t)\right) \cdot \nabla_{x} f^{+}+F \cdot \nabla_{v} f^{+}=-E_{1} \cdot \nabla_{v} f^{+}, \\
& \partial_{t} f^{-}+\left(v-\varepsilon D^{[2]}(t)\right) \cdot \nabla_{x} f^{-}-F \cdot \nabla_{v} f^{-}=E_{1} \cdot \nabla_{v} f^{-} .
\end{aligned}
$$

Since $F$ is small, the characteristics of e.g. (47) should behave as

$$
\mathcal{X}(s) \approx x+(s-t) v+\varepsilon \int_{t}^{s} D^{[2]}(\tau) d \tau, \quad \mathcal{V}(s) \approx v
$$

which is close to a free streaming, at least in case $D^{[2]}$ were not present. Writing $\rho^{ \pm}(t, x)$ as a suitable integral over characteristics, it then turns out that in order 
to derive the necessary estimates for global existence (on higher moments), it is possible to use a rigorous form of (49). In particular, one may verify that

$$
\left|\operatorname{det}\left(\frac{\partial \mathcal{X}}{\partial v}(s)\right)^{-1}\right| \approx|s-t|^{-3} \text { and }\left|\frac{\partial x}{\partial \mathcal{V}}(s)\right| \approx|s-t|,
$$

as is important to transform away the characteristics. The main point to note here is that the term with $D^{[2]}$ drops if we take derivatives in (49) w.r.t. $x$ or $v$, and hence the arguments from [17] can be expected to carry over. Having derived the higher moment bounds

$$
\left.M_{m}(t) \leq C, \quad t \in[0, T], \quad m \in\right] 3, \frac{51}{11}[,
$$

then a standard argument yields the global existence of classical solutions for (VPD).

It should finally be remarked that we did not succeed in generalizing the proofs of global existence for the usual Vlasov-Poisson system that bound the increase in velocity along a characteristic; see 19, 24, 22], and 20 for a recent application. The reason for this is that, when estimating the "ugly" term, an $\ddot{\mathcal{X}}(s)$ will appear, which in our case will lead to the expression $\dot{D}^{[2]}(s)$ that could not be bounded well enough to make the proof work.

\subsection{Local existence}

Similar to the case of the usual Vlasov-Poisson system, cf. 䍩, where this is contained implicitly, an iteration scheme may be set up to yield the local existence of a solution and a criterion when a local solution in fact will be global.

Theorem 3 Suppose $f_{0}^{ \pm}$satisfy 20). Then there exist unique solutions

$$
f^{ \pm} \in C^{1}\left(\left[0, T_{*}\left[\times \mathbb{R}^{3} \times \mathbb{R}^{3}\right)\right.\right.
$$

of (5), (19), (14), and (19) with data $f^{ \pm}(t=0)=f_{0}^{ \pm}$, on a maximal time interval of existence $\left[0, T_{*}[\right.$. If moreover

$$
P=P^{+}+P^{-}, \quad \text { with } \quad P^{ \pm}(t)=\sup \left\{|v|: \exists x \in \mathbb{R}^{3} \exists s \in[0, t]:(x, v) \in \operatorname{supp} f^{ \pm}(s)\right\},
$$

is bounded on $\left[0, T_{*}\left[\right.\right.$, then $T_{*}=\infty$.

We will not go into the proof of this result.

\subsection{Some preliminary estimates}

We first need to derive some a priori bounds. For this we consider a classical solution of the system that exists for times $t \in[0, T]$. Note that all estimates from the previous sections remain valid on any interval where the solution exists. 
Lemma 7 For $t \in[0, T]$ we have

$$
M_{p}(t) \leq C_{T}\left(1+\sup _{s \in[0, t]}\|\nabla U(s)\|_{3+p ; x}^{3+p}\right), \quad p \in[1, \infty[.
$$

In addition,

$$
M_{p}(t) \leq C_{T}\left(1+M_{3\left(\frac{3+2 p}{6+p}\right)}(t)^{\frac{6+p}{3}}\right), \quad p \in[1, \infty[.
$$

Proof: Recalling (37), from (18) and Hölder's inequality it follows that

$$
\begin{aligned}
\frac{d}{d t} M_{p}^{+}(t) & =\iint|v|^{p}\left\{-\left[v+\varepsilon D^{[2]}(t)\right] \cdot \nabla_{x} f^{+}-\nabla U \cdot \nabla_{v} f^{+}\right\} d x d v \\
& =-\iint|v|^{p} \nabla U \cdot \nabla_{v} f^{+} d x d v=p \iint|v|^{p-2}(v \cdot \nabla U) f^{+} d x d v \\
& \leq C\|\nabla U(t)\|_{3+p ; x}\left\|\int|v|^{p-1} f^{+}(t, \cdot, v) d v\right\|_{\frac{3+p}{2+p} ; x} .
\end{aligned}
$$

Now

$$
\left\|\int|v|^{p-1} f^{ \pm}(t, \cdot, v) d v\right\|_{\frac{3+p}{2+p} ; x} \leq C M_{p}^{ \pm}(t)^{\frac{2+p}{3+p}}
$$

by an argument similar to the proof of lemma 3 , and this yields

$$
\left|\frac{d}{d t} M_{p}^{ \pm}(t)\right| \leq C\|\nabla U(t)\|_{3+p ; x} M_{p}(t)^{\frac{2+p}{3+p}} .
$$

Since $M_{p}(\cdot)$ is increasing, it is differentiable a.e. in $t$, with

$$
\frac{d}{d t} M_{p}(t) \leq \sup _{s \in[0, t]}\left|\frac{d}{d t}\left(M_{p}^{+}(s)+M_{p}^{-}(s)\right)\right| \leq C \sup _{s \in[0, t]}\|\nabla U(s)\|_{3+p ; x} M_{p}(t)^{\frac{2+p}{3+p}} .
$$

Integration of this differential inequality gives the claim. Finally, for (51) we observe that by the first part and (42) with $q=3+p$

$$
M_{p}(t) \leq C_{T}+C_{T} \sup _{s \in[0, t]}\|\nabla U(s)\|_{3+p ; x}^{3+p} \leq C_{T}+C_{T} M_{\frac{6 q-9}{3+q}}(t)^{\frac{3+q}{3 q}(3+p)},
$$

and $\frac{6 q-9}{3+q}=3\left(\frac{3+2 p}{6+p}\right)$.

\subsection{Estimates for higher moments}

For $R>0$ choose a radially symmetric function $\chi_{R} \in C_{0}^{\infty}\left(\mathbb{R}^{3}\right)$ with $\chi_{R}(x) \in[0,1]$ for $x \in \mathbb{R}^{3}, \chi_{R}(x)=1$ for $|x| \leq R$, and $\chi(x)=0$ for $|x| \geq 2 R$. Correspondingly we decompose the electric field $E(t, x)$ from (26) as

$$
E(t, x)=E_{1}(t, x)+F(t, x),
$$


with

$$
\begin{aligned}
E_{1}(t, x) & =\int \chi(x-y) \frac{(x-y)}{|x-y|^{3}}\left(\rho^{+}(t, y)-\rho^{-}(t, y)\right) d y \\
& =-\left(\chi \nabla \frac{1}{|x|}\right) *\left(\rho^{+}(t)-\rho^{-}(t)\right)(x) .
\end{aligned}
$$

Some useful estimates on $E_{1}$ and $F$ are stated in lemma 15 below. Then we write the Vlasov equations $(18)$ and (19) in the form (47) and (48). This can be used to derive a representation formula for $\rho^{ \pm}(t, x)$, and for simplicity we will consider only $\rho^{+}(t, x)$. We fix $x, v \in \mathbb{R}^{3}$ and $t \in[0, T]$, and denote $(X(s), V(s))=$ $(X(s ; x, v), V(s ; x, v))$ for $s \in[0, t]$ the solution of the characteristic system

$$
\left(\begin{array}{c}
\dot{X}(s) \\
\dot{V}(s)
\end{array}\right)=\left(\begin{array}{c}
-V(s)-\varepsilon D^{[2]}(t-s) \\
-F(t-s, X(s))
\end{array}\right), \quad\left(\begin{array}{c}
X(0) \\
V(0)
\end{array}\right)=\left(\begin{array}{c}
x \\
v
\end{array}\right)
$$

associated with (47). Since

$$
\begin{aligned}
\frac{\partial}{\partial s} & {\left[f^{+}(t-s, X(s), V(s))\right] } \\
= & -\partial_{t} f^{+}(t-s, X(s), V(s))-\left[V(s)+\varepsilon D^{[2]}(t-s)\right] \cdot \partial_{X} f^{+}(t-s, X(s), V(s)) \\
& -F(t-s, X(s)) \cdot \partial_{V} f^{+}(t-s, X(s), V(s)) \\
= & E_{1}(t-s, X(s)) \cdot \partial_{V} f^{+}(t-s, X(s), V(s))
\end{aligned}
$$

by (47), it follows through integrating $\int_{0}^{t} d s(\ldots)$ and $\int d v(\ldots)$ that

$$
\begin{aligned}
\rho^{+}(t, x)= & \int d v f_{0}^{+}(X(t), V(t)) \\
& -\int_{0}^{t} d s \int d v E_{1}(t-s, X(s)) \cdot \partial_{V} f^{+}(t-s, X(s), V(s)) \\
= & \int d v f_{0}^{+}(X(t), V(t))-\int_{0}^{t} d s \int d v \operatorname{div}_{V}\left[E_{1} f^{+}\right](t-s, X(s), V(s)),
\end{aligned}
$$

where $f_{0}^{+}=f^{+}(t=0)$, and $\left[E_{1} f^{+}\right](\tau, X, V)=E_{1}(\tau, X) f^{+}(\tau, X, V)$; note that the dependence on $x$ and $v$ in (55) enters via $X(s)$ and $V(s)$. To rewrite (55) appropriately, define

$$
G(X, V)=\left[E_{1} f^{+}\right](t-s, X, V) \text { and } \quad \tilde{G}(x, v)=G(X(s ; x, v), V(s ; x, v)) .
$$

By lemma 16 below we then have $G(X, V)=\tilde{G}(x(s ; X, V), v(s ; X, V))$, and consequently

$$
\operatorname{div}_{V} G=\operatorname{div}_{x}\left(\frac{\partial x}{\partial V} \cdot \tilde{G}\right)-\operatorname{div}_{x}\left(\frac{\partial x}{\partial V}\right) \cdot \tilde{G}+\sum_{i, j=1}^{3}\left(\frac{\partial \tilde{G}_{i}}{\partial v_{j}}\right)\left(\frac{\partial v_{j}}{\partial V_{i}}\right),
$$


where $\operatorname{div}_{x}\left(\frac{\partial x}{\partial V}\right) \cdot \tilde{G}=\sum_{i, j=1}^{3} \tilde{G}_{i} \frac{\partial}{\partial x_{j}}\left(\frac{\partial x_{j}}{\partial V_{i}}\right)$. Utilizing this in (55) and integrating by parts w.r.t. $v$ yields

$$
\begin{aligned}
\rho^{+}(t, x)= & \int d v f_{0}^{+}(X(t), V(t))-\operatorname{div}_{x} \int_{0}^{t} d s \int d v\left(\frac{\partial x}{\partial V} \cdot \tilde{G}\right) \\
& +\int_{0}^{t} d s \int d v\left[\operatorname{div}_{x}\left(\frac{\partial x}{\partial V}\right) \cdot \tilde{G}+\operatorname{div}_{v}\left(\frac{\partial v}{\partial V}\right) \cdot \tilde{G}\right] \\
=: & \phi_{0}^{+}(t, x)-\operatorname{div}_{x} \Gamma^{+}(t, x)+R^{+}(t, x) .
\end{aligned}
$$

Similarly, we have

$$
\rho^{-}(t, x)=\phi_{0}^{-}(t, x)-\operatorname{div}_{x} \Gamma^{-}(t, x)+R^{-}(t, x),
$$

with the corresponding functions $\phi_{0}^{-}, \Gamma^{-}$, and $R^{-}$

Next we derive some estimates on $\phi_{0}^{+}, \Gamma^{+}$, and $R^{+}$.

Lemma 8 For $t \in[0, T]$ we have

$$
\left\|\phi_{0}^{+}(t)\right\|_{\frac{3+m}{3} ; x} \leq C_{T} \quad(m>0) \quad \text { and } \quad\left\|\phi_{0}^{+}(t)\right\|_{3\left(\frac{3+m}{6+m}\right) ; x} \leq C_{T} \quad(m \geq 3) .
$$

Proof: We can apply corollary 7 below with $s=t$ and $\tau=0$ to obtain the first bound. Concerning the second, note that $m \geq 3$ implies $3\left(\frac{3+m}{6+m}\right) \leq \frac{3+m}{3}$. Whence it suffices to bound the support of $x \mapsto \phi_{0}^{+}(t, x)=\int d v f_{0}^{+}(X(t ; x, v), V(t ; x, v))$. To do so, recall from (20) that $f_{0}^{+}(\bar{x}, \bar{v})=0$ for $|\bar{x}| \geq r_{0}$ or $|\bar{v}| \geq r_{0}$. From the proof of corollary 7 we know $|V(t)-v| \leq C_{1}, C_{1}$ depending only on $T$. Thus $\frac{\partial}{\partial s} \mid X(s)-$ $x|\leq| V(s)+\varepsilon D^{[2]}(t-s) \mid \leq C(1+|v|)$ by (54) and theorem 2(c), whence $|X(t)-x| \leq$ $C_{2}(1+|v|)$. Then, if $|x| \geq C_{2}\left(1+C_{1}+r_{0}\right)+r_{0}=: r_{1}$ and $|V(t)| \leq r_{0}$, we have $|v| \leq$ $|V(t)-v|+|V(t)| \leq C_{1}+r_{0}$ and therefore $|X(t)| \geq|x|-|X(t)-x| \geq r_{0}$. This yields $f_{0}^{+}(X(t), V(t))=0$, and thus $\phi_{0}^{+}(t, x)=0$ for $|x| \geq r_{1}$ and $t \in[0, T]$.

Next we turn to bound $\Gamma^{+}(t, x)=\int_{0}^{t} d s \int d v\left(\frac{\partial x}{\partial V} \cdot \tilde{G}\right)$.

Lemma 9 For $t \in[0, T]$ and any $\left.\left.t_{0} \in\right] 0, T\right]$ we have

$$
\left\|\Gamma^{+}(t)\right\|_{3+m ; x} \leq C_{T} t_{0}^{\frac{m-3}{6-m}}\left(1+M_{m}(t)^{\frac{9}{(6-m)(3+m)}}\right)+C_{T}\left(1+\left|\ln t_{0}\right|\right)\left(1+M_{m}(t)^{\frac{1}{3+m}}\right),
$$

with $3<m<\frac{51}{11}$. Here $C_{T}$ does not depend on $t_{0}$. Moreover,

$$
\left\|\Gamma^{+}(t)\right\|_{3+m ; x} \leq C_{T} t^{\frac{m-3}{6-m}}\left(1+M_{m}(t)^{\frac{9}{(6-m)(3+m)}}\right), \quad t \in[0, T] .
$$

Proof: We first note that

$$
\left|\Gamma^{+}(t, x)\right| \leq C_{T} \int_{0}^{t} d s s \int d v\left|\left[E_{1} f^{+}\right](t-s, X(s ; x, v), V(s ; x, v))\right|,
$$


in view of the second estimate in (67) below. Next we observe that due to lemma 2 and the first estimate in (67) we have, with $\frac{1}{r}+\frac{1}{r^{\prime}}=1$,

$$
\begin{aligned}
& \int d v\left|\left[E_{1} f^{+}\right](t-s, X(s), V(s))\right| \\
& \leq \sup _{\tau \in[0, T]}\left\|f^{+}(\tau)\right\|_{\infty ; x v}^{\frac{r^{\prime}-1}{r^{\prime}}}\left(\int d v\left|E_{1}(t-s, X(s))\right|^{r}\right)^{\frac{1}{r}}\left(\int d v f^{+}(t-s, X(s), V(s))\right)^{\frac{1}{r^{\prime}}} \\
& \leq C_{T} s^{-\frac{3}{r}}\left(\int d X\left|E_{1}(t-s, X)\right|^{r}\right)^{\frac{1}{r}}\left(\int d v f^{+}(t-s, X(s), V(s))\right)^{\frac{1}{r^{\prime}}} \\
& \leq C_{T} s^{-\frac{3}{r}} \sup _{\tau \in[0, T]}\left\|E_{1}(\tau)\right\|_{r ; x}\left(\int d v f^{+}(t-s, X(s), V(s))\right)^{\frac{1}{r^{\prime}}} .
\end{aligned}
$$

In (59) we then split $\int_{0}^{t} d s=\int_{0}^{t_{0}} d s+\int_{t_{0}}^{t} d s$ with $\left.\left.t_{0} \in\right] 0, t\right]$.

To bound the first term, we choose $r^{\prime}=6-m<3$, whence $r=\frac{6-m}{5-m}>\frac{3}{2}$. Then with $p=\frac{6 m-9}{6-m}>1$ we find $\frac{3+m}{r^{\prime}}=\frac{3+p}{3}$. Moreover, $1 \leq r<\frac{15}{4}$ by the choice of $m$. Hence by lemma 15 and corollary 0

$$
\begin{aligned}
& \left\|\int_{0}^{t_{0}} d s s \int d v\left|\left[E_{1} f^{+}\right](t-s, X(s ; \cdot, v), V(s ; \cdot, v))\right|\right\|_{3+m ; x} \\
& \leq C_{T} t_{0}^{2-\frac{3}{r}}\left(\sup _{\tau \in[0, T]}\left\|E_{1}(\tau)\right\|_{r ; x}\right) \\
& \quad \times \sup _{\tau \in[0, t]}\left[\int d x\left(\int d v f^{+}(t-\tau, X(\tau), V(\tau))\right)^{\frac{3+m}{r^{\prime}}}\right]^{\frac{1}{3+m}} \\
& \leq C_{T} t_{0}^{2-\frac{3}{r}}\left(1+M_{p}(t)^{\frac{1}{3+m}}\right)
\end{aligned}
$$

note that Jensen's inequality has been used for the first estimate. Utilizing the relation $3\left(\frac{3+2 p}{6+p}\right)=m$ and 51 , we can bound $M_{p}(t)$ by means of $M_{m}(t)$, as

$$
M_{p}(t) \leq C_{T}\left(1+M_{m}(t)^{\frac{6+p}{3}}\right)=C_{T}\left(1+M_{m}(t)^{\frac{9}{6-m}}\right) .
$$

Thus we have shown that

$$
\begin{gathered}
\left\|\int_{0}^{t_{0}} d s s \int d v\left|\left[E_{1} f^{+}\right](t-s, X(s ; \cdot, v), V(s ; \cdot, v))\right|\right\|_{3+m ; x} \\
\leq C_{T} t_{0}^{2-\frac{3}{r}}\left(1+M_{m}(t)^{\frac{9}{(6-m)(3+m)}}\right) .
\end{gathered}
$$

As far as the second part of the integral is concerned, we now make use of (60) with $r^{\prime}=3$ and $r=\frac{3}{2}$. Then

$$
\left\|\int_{t_{0}}^{t} d s s \int d v\left|\left[E_{1} f^{+}\right](t-s, X(s ; \cdot, v), V(s ; \cdot, v))\right|\right\|_{3+m ; x}
$$




$$
\begin{aligned}
& \leq C_{T}\left(1+\left|\ln t_{0}\right|\right)\left(\sup _{\tau \in[0, T]}\left\|E_{1}(\tau)\right\|_{\frac{3}{2} ; x}\right) \\
& \quad \times \sup _{\tau \in[0, t]}\left[\int d x\left(\int d v f^{+}(t-\tau, X(\tau), V(\tau))\right)^{\frac{3+m}{3}}\right]^{\frac{1}{3+m}} \\
& \leq C_{T}\left(1+\left|\ln t_{0}\right|\right)\left(1+M_{m}(t)^{\frac{1}{3+m}}\right)
\end{aligned}
$$

again by lemma 15 and corollary 0 . Summarizing (61) and (62), we see that the first asserted estimate holds. To verify (58) it sufficient to follow the argument just elaborated and to note that for $t_{0}=t$ the contribution of the $\int_{t_{0}}^{t} d s(\ldots)$-part of (59) drops out, whence we simply use (61) for $t_{0}=t$.

Finally we need to consider

$$
R^{+}(t, x)=\int_{0}^{t} d s \int d v\left[\operatorname{div}_{x}\left(\frac{\partial x}{\partial V}\right) \cdot \tilde{G}+\operatorname{div}_{v}\left(\frac{\partial v}{\partial V}\right) \cdot \tilde{G}\right]
$$

in (56).

Lemma 10 For $t \in[0, T]$ we have

$$
\left\|R^{+}(t)\right\|_{3\left(\frac{3+m}{6+m}\right) ; x} \leq C_{T},
$$

with $m \in\left[0, \frac{147}{16}\right]$.

Proof: Using (68) below, and (60) with $r^{\prime}=\frac{13}{9}$ and $r=\frac{13}{4}$, we estimate

$$
\begin{aligned}
\left|R^{+}(t, x)\right| & \leq C_{T} \int_{0}^{t} d s \int d v\left|\left[E_{1} f^{+}\right](t-s, X(s ; x, v), V(s ; x, v))\right| \\
& \leq C_{T}\left(\sup _{\tau \in[0, T]}\left\|E_{1}(\tau)\right\|_{\frac{13}{4} ; x}\right) \int_{0}^{t} d s s^{-\frac{12}{13}}\left(\int d v f^{+}(t-s, X(s), V(s))\right)^{\frac{9}{13}} \\
& \leq C_{T} \int_{0}^{t} d s s^{-\frac{12}{13}}\left(\int d v f^{+}(t-s, X(s), V(s))\right)^{\frac{9}{13}}
\end{aligned}
$$

the latter according to lemma 15. Hence due to corollary 7, with $p$ determined through $\frac{3+p}{3}=\frac{27}{13}\left(\frac{3+m}{6+m}\right)$,

$$
\begin{aligned}
\left\|R^{+}(t)\right\|_{3\left(\frac{3+m}{6+m}\right) ; x} & \leq C_{T} \sup _{\tau \in[0, t]}\left[\int d x\left(\int d v f^{+}(t-\tau, X(\tau), V(\tau))\right)^{\frac{2 \tau}{13}\left(\frac{3+m}{6+m}\right)}\right]^{\frac{6+m}{3(3+m)}} \\
& \leq C_{T}\left(1+M_{p}(t)^{\frac{6+m}{3(3+m)}}\right)=C_{T}\left(1+M_{3(\alpha-1)}(t)^{\frac{6+m}{3(3+m)}}\right)
\end{aligned}
$$


where $\alpha=\frac{27}{13}\left(\frac{3+m}{6+m}\right)$. Since $0 \leq 3(\alpha-1)=\frac{3}{13}\left(\frac{3+14 m}{6+m}\right) \leq 2$ by choice of $m$, the claim follows from lemma 2 .

The foregoing estimates, and analogous ones for $\phi_{0}^{-}, \Gamma^{-}$, and $R^{-}$, can be put together to yield the following result.

Lemma 11 For $t \in[0, T]$ and $m \in] 3, \frac{51}{11}[$ we have

$$
\begin{aligned}
\|\nabla U(t)\|_{3+m ; x} \leq & C_{T} t_{0}^{\frac{m-3}{6-m}}\left(1+M_{m}(t)^{\frac{9}{(6-m)(3+m)}}\right) \\
& +C_{T}\left(1+\left|\ln t_{0}\right|\right)\left(1+M_{m}(t)^{\frac{1}{3+m}}\right),
\end{aligned}
$$

with $\left.\left.t_{0} \in\right] 0, t\right]$ being arbitrary. Here $C_{T}$ does not depend on $t_{0}$. Moreover,

$$
\|\nabla U(t)\|_{3+m ; x} \leq C_{T} t^{\frac{m-3}{6-m}}\left(1+M_{m}(t)^{\frac{9}{(6-m)(3+m)}}\right), \quad t \in[0, T] .
$$

Proof: Due to (26), (56), and (57) we may write

$$
\begin{aligned}
\nabla U(t, x)= & -\left(\nabla \frac{1}{|x|}\right) *\left(\left[\phi_{0}^{+}(t)-\phi_{0}^{-}(t)\right]+\left[R^{+}(t)-R^{-}(t)\right]\right. \\
& \left.+\operatorname{div}_{x}\left[\Gamma^{-}(t)-\Gamma^{+}(t)\right]\right)(x) .
\end{aligned}
$$

Therefore lemma 4 implies for $m \in[0, \infty[$ that

$$
\begin{aligned}
\|\nabla U(t)\|_{3+m ; x} \leq & C\left(\left\|\phi_{0}^{+}(t)\right\|_{3\left(\frac{3+m}{6+m}\right) ; x}+\left\|\phi_{0}^{-}(t)\right\|_{3\left(\frac{3+m}{6+m}\right) ; x}\right. \\
& \left.+\left\|R^{+}(t)\right\|_{3\left(\frac{3+m}{6+m}\right) ; x}+\left\|R^{-}(t)\right\|_{3\left(\frac{3+m}{6+m}\right) ; x}\right) \\
+ & C\left(\left\|\Gamma^{+}(t)\right\|_{3+m ; x}+\left\|\Gamma^{-}(t)\right\|_{3+m ; x}\right) .
\end{aligned}
$$

Due to lemmas 8, 9, and 10 we thus obtain the first desired estimate. Concerning (64), we rather apply (58) than the first estimate from lemma 9 .

This in particular can be used to derive a short time bound on $M_{m}(t)$.

Corollary 4 For $m \in] 3, \frac{51}{11}\left[\right.$ there exist $\left.\left.t_{1} \in\right] 0, T\right]$ and $C_{1}>0$ (both depending on T) such that

$$
M_{m}(t) \leq C_{1}, \quad t \in\left[0, t_{1}\right]
$$

Proof : Combining (52) with (64) and observing $t^{\frac{m-3}{6-m}} \leq C_{T}$ yields

$$
\begin{aligned}
\frac{d}{d t} M_{m}(t) & \leq C \sup _{s \in[0, t]}\|\nabla U(s)\|_{3+m ; x} M_{m}(t)^{\frac{2+m}{3+m}} \\
& \leq C_{T}\left(1+M_{m}(t)^{\frac{9}{(6-m)(3+m)}}\right) M_{m}(t)^{\frac{2+m}{3+m}}
\end{aligned}
$$


Integration of this differential inequality gives a local bound on $M_{m}(t)$, that, however, fails to extend to all of $[0, T]$ due to $\frac{9}{(6-m)(3+m)}+\frac{2+m}{3+m}=\frac{7-m}{6-m}>1$.

Corollary 5 For $t \in[0, T]$ and $m \in] 3, \frac{51}{11}[$ we have

$$
\|\nabla U(t)\|_{3+m ; x} \leq C_{T}\left(1+\left|\ln M_{m}(t)\right|\right)\left(1+M_{m}(t)^{\frac{1}{3+m}}\right) .
$$

Proof: Note that we may assume $M_{m}(t) \geq 1$ for $t \in[0, T]$, since otherwise $M_{m}(t)$ simply can be replaced by $M_{m}(t)+1$. Set

$$
t_{0}=t_{1} M_{m}(t)^{\frac{1}{3-m}} \leq t_{1}
$$

in lemma 11, with $t_{1}$ from corollary 4 . If $t \in\left[t_{1}, T\right]$, then $t_{0} \leq t$, and therefore $\frac{1}{3-m}\left(\frac{m-3}{6-m}\right)+\frac{9}{(6-m)(3+m)}=\frac{1}{3+m}$ shows that (65) holds for $t \in\left[t_{1}, T\right]$. On the other hand, if $t \in\left[0, t_{1}\right]$, then $M_{m}(t) \leq C_{1}$ and (64) imply $\|\nabla U(t)\|_{3+m ; x} \leq C_{2}$ for some $C_{2}>0$. Hence (65) holds as well in this case if we choose $C_{T} \geq C_{2}$.

Theorem 4 For $t \in[0, T]$ and $m \in] 3, \frac{51}{11}[$ we have

$$
M_{m}(t) \leq C_{T}
$$

Proof : By 52 and due to corollary 5 we see that

$$
\begin{aligned}
\frac{d}{d t} M_{m}(t) & \leq C \sup _{s \in[0, t]}\|\nabla U(s)\|_{3+m ; x} M_{m}(t)^{\frac{2+m}{3+m}} \leq C_{T}\left(1+\left|\ln M_{m}(t)\right|\right)\left(1+M_{m}(t)\right) \\
& \leq C_{T}\left(1+\left|\ln M_{m}(t)\right| M_{m}(t)\right)
\end{aligned}
$$

Integration of this differential inequality yields the claimed estimate.

Corollary 6 For $t \in[0, T]$ we have

$$
\left.\left\|\rho^{ \pm}(t)\right\|_{p ; x} \leq C_{T}, \quad p \in\right] 2, \frac{28}{11}[.
$$

Proof: This is a consequence of (41) and theorem 4 , since $m=3(p-1) \in] 3, \frac{51}{11}[$ corresponds to $\left.p=\frac{3+m}{3} \in\right] 2, \frac{28}{11}[$. 


\subsection{Global existence of solutions}

We start with some preliminary (well-known) observations. Recall the definition of $P^{ \pm}(t)$ from (50), and also that $\left[0, T_{*}\right.$ [ is the maximal interval of existence, cf. theorem 3 .

\section{Lemma 12 We have}

$$
P^{ \pm}(t) \leq P^{ \pm}(0)+\int_{0}^{t}\|\nabla U(s)\|_{\infty ; x} d s, \quad t \in\left[0, T_{*}[.\right.
$$

Proof: Assume e.g. $(x, v) \in \operatorname{supp} f^{+}(s)$ for some $x \in \mathbb{R}^{3}$ and $s \in[0, t]$. From the proof of lemma 2 we have $f^{+}(s, x, v)=f_{0}^{+}(\mathcal{X}(0 ; s, x, v), \mathcal{V}(0 ; s, x, v))$, with $(\mathcal{X}, \mathcal{V})$ the characteristics from (38). This means that $(x, v)=\left(\mathcal{X}\left(s ; 0, x_{0}, v_{0}\right), \mathcal{V}\left(s ; 0, x_{0}, v_{0}\right)\right)$ for $\left(x_{0}, v_{0}\right) \in \operatorname{supp} f_{0}^{+}$. Hence

$$
|v| \leq\left|v_{0}\right|+\left|\int_{0}^{s} \dot{\mathcal{V}}\left(\tau ; 0, x_{0}, v_{0}\right) d \tau\right| \leq P^{+}(0)+\int_{0}^{t}\|\nabla U(\tau)\|_{\infty ; x} d \tau
$$

by the characteristic equation for $\mathcal{V}$.

Next we need to derive a bound on $\|\nabla U(t)\|_{\infty ; x}$.

Lemma 13 For $\alpha>\frac{3}{2}$ we have

$$
\|\nabla U(t)\|_{\infty ; x} \leq C\left(\sum_{j= \pm}\left\|\rho^{j}(t)\right\|_{\infty ; x}\right)^{1-\frac{\alpha^{\prime}}{3}}\left(\sum_{j= \pm}\left\|\rho^{j}(t)\right\|_{\alpha^{\prime} ; x}\right)^{\frac{\alpha^{\prime}}{3}}, \quad t \in\left[0, T_{*}[,\right.
$$

where $\frac{1}{\alpha}+\frac{1}{\alpha^{\prime}}=1$. The constant $C$ depends only on $\alpha$.

Proof: With $R>0$ we estimate from 26

$$
\begin{aligned}
|\nabla U(t, x)| \leq & \left.\int_{|y-x| \leq R} \frac{d y}{|x-y|^{2}}\left(\rho^{+}(t, y)+\rho^{-}(t, y)\right)\right) \\
& \left.+\int_{|y-x| \geq R} \frac{d y}{|x-y|^{2}}\left(\rho^{+}(t, y)+\rho^{-}(t, y)\right)\right) \\
\leq & C\left(\sum_{j= \pm}\left\|\rho^{j}(t)\right\|_{\infty ; x}\right) R+\left(\int_{|y-x| \geq R} \frac{d y}{|x-y|^{2 \alpha}}\right)^{\frac{1}{\alpha}}\left(\sum_{j= \pm}\left\|\rho^{j}(t)\right\|_{\alpha^{\prime} ; x}\right) \\
\leq & C\left(\sum_{j= \pm}\left\|\rho^{j}(t)\right\|_{\infty ; x}\right) R+C\left(\sum_{j= \pm}\left\|\rho^{j}(t)\right\|_{\alpha^{\prime} ; x}\right) R^{\frac{3}{\alpha}-2} .
\end{aligned}
$$

Choosing the optimal $R$ yields the claim. 
Lemma 14 We have

$$
\left\|\rho^{ \pm}(t)\right\|_{\infty ; x} \leq C P^{ \pm}(t)^{3}, \quad t \in\left[0, T_{*}[,\right.
$$

with $C$ depending only on the data.

Proof : By definition of $P^{+}(t)$ and bounding $\left\|f^{ \pm}(t)\right\|_{\infty ; x v}$ as in lemma 2, it follows that

$$
\rho^{+}(t, x)=\int_{|v| \leq P^{+}(t)} f^{+}(t, x, v) d v \leq C\left\|f^{ \pm}(t)\right\|_{\infty ; x v} P^{+}(t)^{3} \leq C P^{+}(t)^{3},
$$

as was to be shown.

Using the criterion from theorem 3 and by means of corollary 6 we are finally going to complete the proof of theorem 1 .

Proof of theorem 11: Assume $T_{*}<\infty$ in theorem 3. All the estimates on the moments remain valid if $[0, T]$ is replaced by $\left[0, T_{*}[\right.$, since in the constants only terms of the form $C T_{*}, T_{*}^{\alpha}$, and $e^{C T_{*}}$ do enter. In particular,

$$
\left\|\rho^{ \pm}(t)\right\|_{\frac{27}{11} ; x} \leq C, \quad t \in\left[0, T_{*}[,\right.
$$

by corollary 6 , where here and below the various constants $C$ depend on $T_{*}$. Choosing $\alpha=\frac{27}{16}>\frac{3}{2}$, which corresponds to $\alpha^{\prime}=\frac{27}{11}$, we deduce from lemmas 12, 13, and 14 that

$$
\begin{aligned}
P^{+}(t) & \leq P^{+}(0)+C \int_{0}^{t} d s\left(\sum_{j= \pm}\left\|\rho^{j}(s)\right\|_{\infty ; x}\right)^{\frac{2}{11}}\left(\sum_{j= \pm}\left\|\rho^{j}(s)\right\|_{\frac{27}{11} ; x}\right)^{\frac{9}{11}} \\
& \leq P^{+}(0)+C \int_{0}^{t} d s\left(P^{+}(s)^{3}+P^{-}(s)^{3}\right)^{\frac{2}{11}} .
\end{aligned}
$$

This implies

$$
P(t) \leq P(0)+C \int_{0}^{t} P(s)^{\frac{6}{11}} d s, \quad t \in\left[0, T_{*}[,\right.
$$

and hence the boundedness of $P$ on $\left[0, T_{*}[\right.$.

We remark that we did not try to reduce the exponent $\frac{6}{11}$ so as to obtain the optimal power of $P$.

\subsection{Some technical lemmas}

Lemma 15 Define $E_{1}$ and $F$ as in (55). Then we have $\left\|E_{1}(t)\right\|_{p ; x} \leq C$ for $t \in[0, T]$ and $p \in\left[1, \frac{15}{4}[\right.$, as well as

$$
\|F(t)\|_{\infty ; x}+\|\nabla F(t)\|_{\infty ; x}+\left\|D^{2} F(t)\right\|_{\infty ; x} \leq C R^{-2}, \quad t \in[0, T] .
$$


Proof: From (53) and Young's inequality [21, p. 29] with $\frac{1}{q}+\frac{1}{r}=1+\frac{1}{p}$ we obtain

$$
\left\|E_{1}(t)\right\|_{p ; x}=\left\|\left(\chi \nabla \frac{1}{|x|}\right) *\left(\rho^{+}(t)-\rho^{-}(t)\right)\right\|_{p ; x} \leq\left\|\chi \nabla \frac{1}{|x|}\right\|_{q ; x}\left\|\rho^{+}(t)-\rho^{-}(t)\right\|_{r ; x} .
$$

We have $\chi(\cdot) \frac{x}{|x|^{3}} \in L^{q}\left(\mathbb{R}^{3}\right)$ for $q \in\left[1, \frac{3}{2}\left[\right.\right.$ and $\left\|\rho^{ \pm}(t)\right\|_{r ; x} \leq C$ for $r \in\left[1, \frac{5}{3}\right]$ due to theorem 2(a). Combining those values for $q$ and $r$, we see that we need to have $p \in\left[1, \frac{15}{4}[\right.$. The bounds in (66) are obtained by observing that

$$
F(t, x)=\int_{|x-y| \geq R}(1-\chi(x-y)) \frac{(x-y)}{|x-y|^{3}}\left(\rho^{+}(t, y)-\rho^{-}(t, y)\right) d y
$$

where $\chi \in C_{0}^{\infty}\left(\mathbb{R}^{3}\right)$, and moreover $\int \rho^{ \pm}(t, y) d y=\iint f^{ \pm}(t, y, v) d y d v \leq C$.

Lemma 16 For fixed $t \in] 0, T]$ and $s \in[0, t]$ consider the map

$$
Z(s): \quad \mathbb{R}^{6} \ni(x, v) \mapsto(X(s ; x, v), V(s ; x, v))=(X(s), V(s)) \in \mathbb{R}^{6},
$$

where $(X(s), V(s))$ is the solution of the characteristic system (54), i.e.

$$
\left(\begin{array}{c}
\dot{X}(s) \\
\dot{V}(s)
\end{array}\right)=\left(\begin{array}{c}
-V(s)-\varepsilon D^{[2]}(t-s) \\
-F(t-s, X(s))
\end{array}\right), \quad\left(\begin{array}{c}
X(0) \\
V(0)
\end{array}\right)=\left(\begin{array}{c}
x \\
v
\end{array}\right) .
$$

Then $Z(s)$ is a volume-preserving diffeomorphism, and

$$
\left|\operatorname{det}\left(\frac{\partial X}{\partial v}(s)\right)^{-1}\right| \leq C s^{-3}, \quad\left|\frac{\partial x}{\partial V}(s)\right| \leq C s,
$$

as well as

$$
\left|\frac{\partial}{\partial x_{i}}\left(\frac{\partial x_{j}}{\partial V_{k}}(s)\right)\right|+\left|\frac{\partial}{\partial v_{i}}\left(\frac{\partial v_{j}}{\partial V_{k}}(s)\right)\right| \leq C, \quad 1 \leq i, j, k \leq 3,
$$

if $R>0$ is chosen sufficiently large (depending on $T$ ). Here

$$
Z(s)^{-1}:(X, V) \mapsto(x, v)=(x(s ; X, V), v(s ; X, V))
$$

is the inverse of $Z(s)$, and the constants $C$ do depend only on $T$, but not on $(t, s, x, v)$.

Proof: As the right-hand side of the characteristic system has divergence div $=$ $\operatorname{div}_{(X, V)}=0$, the first claim follows; cf. also remark 2. Moreover, we have

$$
\begin{aligned}
& \left|\frac{\partial X}{\partial v}(s)+s \operatorname{Id}\right| \leq C R^{-2} s^{3}, \quad\left|\frac{\partial V}{\partial v}(s)-\mathrm{Id}\right| \leq C R^{-2} s^{2}, \\
& \left|\frac{\partial X}{\partial x}(s)-\mathrm{Id}\right| \leq C R^{-2} s^{2}, \quad\left|\frac{\partial V}{\partial x}(s)\right| \leq C R^{-2} s,
\end{aligned}
$$


where Id denotes the unit matrix in $\mathbb{R}^{3}$. E.g. to validate the estimate on the $v$ derivatives one can introduce, following [3], the function $\phi(s)=\frac{\partial X}{\partial v}(s)+s \operatorname{Id}$ and calculate that $\phi(0)=0, \dot{\phi}(s)=-\frac{\partial V}{\partial v}(s)+\mathrm{Id}, \dot{\phi}(0)=0$, as well as

$$
\ddot{\phi}(s)=\frac{\partial F}{\partial x}(t-s, X(s))[\phi(s)-s \mathrm{Id}] .
$$

Here the important observation is that

$$
\begin{aligned}
\dot{\phi}(s) & =\frac{\partial}{\partial s}\left(\frac{\partial X}{\partial v}(s)\right)+\mathrm{Id}=\frac{\partial}{\partial v} \dot{X}(s)+\operatorname{Id}=\frac{\partial}{\partial v}\left(-V(s)-\varepsilon D^{[2]}(t-s)\right)+\mathrm{Id} \\
& =-\frac{\partial V}{\partial v}(s)+\mathrm{Id},
\end{aligned}
$$

since the term with $\varepsilon D^{[2]}(t-s)$ simply drops through the $v$-derivative, and thus the same general argument can be used as in the case without $D^{[2]}$. Then we may write $\phi(s)=\int_{0}^{s}(s-\tau) \ddot{\phi}(\tau) d \tau$ and utilize (66) to derive (69) by means of a Gronwall argument, whereas (70) is obtained in the same way using the function $\phi(s)=\frac{\partial X}{\partial x}(s)-$ Id instead.

Then

$$
\left|\operatorname{det}\left(\frac{\partial X}{\partial v}(s)\right)\right|=s^{3}\left|\operatorname{det}\left(-s^{-1}\left[\frac{\partial X}{\partial v}(s)+s \mathrm{Id}\right]+\mathrm{Id}\right)\right|
$$

together with (69) and the continuity of the map $A \mapsto|\operatorname{det}(A)|$ at $A=\mathrm{Id}$ yields that for $s \in[0, T]$ and $R>0$ large enough,

$$
\left|\operatorname{det}\left(\frac{\partial X}{\partial v}(s)\right)\right| \geq \frac{1}{2} s^{3},
$$

and this proves the first estimate in (67). As what concerns the bound on $\left|\frac{\partial x}{\partial V}(s)\right|$, employing the chain rule it follows that

$$
\begin{aligned}
& \frac{\partial x}{\partial V}(s)=-\left(\frac{\partial x}{\partial X}(s)\right)\left(\frac{\partial X}{\partial v}(s)\right)\left(\frac{\partial V}{\partial v}(s)\right)^{-1} \\
& \frac{\partial x}{\partial X}(s)=\left[\operatorname{Id}-\left(\frac{\partial x}{\partial V}(s)\right)\left(\frac{\partial V}{\partial x}(s)\right)\right]\left(\frac{\partial X}{\partial x}(s)\right)^{-1} .
\end{aligned}
$$

Choosing $R>0$ sufficiently large, we find from (69) and (70) that

$$
\left|\left(\frac{\partial V}{\partial v}(s)\right)^{-1}\right|+\left|\left(\frac{\partial X}{\partial x}(s)\right)^{-1}\right| \leq C,
$$

whence by (69) and (70),

$$
\left|\frac{\partial x}{\partial V}(s)\right| \leq C s\left|\frac{\partial x}{\partial X}(s)\right|, \quad\left|\frac{\partial x}{\partial X}(s)\right| \leq C\left(1+R^{-2} s\left|\frac{\partial x}{\partial V}(s)\right|\right)
$$


and this gives $\left|\frac{\partial x}{\partial V}(s)\right| \leq C s$, for $R>0$ large enough.

Finally, the estimates on the second derivatives in (68) are more tedious, but verified in a similar way.

Corollary 7 If $(X(s), V(s))$ is a characteristic curve, cf. lemma 10, and $s, \tau \in$ $[0, T]$, then for $p \in] 0, \infty[$

$\left\|\int f^{ \pm}(\tau, X(s ; \cdot, v), V(s ; \cdot, v)) d v\right\|_{\frac{3+p}{3} ; x} \leq C_{T}\left\|f^{ \pm}(\tau)\right\|_{\infty ; x v}^{\frac{p}{3+p}}\left(M_{0}(\tau)^{\frac{3}{3+p}}+M_{p}(\tau)^{\frac{3}{3+p}}\right)$,

with $M_{p}(\cdot)$ from (37).

Proof : Utilizing lemma 3 with $f(x, v)=f^{ \pm}(\tau, X(s ; x, v), V(s ; x, v))$, it follows that the left-hand side is bounded by

$$
C\left\|f^{ \pm}(\tau)\right\|_{\infty ; x v}^{\frac{p}{3+p}}\left(\iint|v|^{p} f^{ \pm}(\tau, X(s ; x, v), V(s ; x, v)) d x d v\right)^{\frac{3}{3+p}} .
$$

From the characteristic equation for $V(s)$ we obtain that $\frac{\partial}{\partial s}|V(s)-v| \leq\|F\|_{\infty ; x t} \leq$ $C$, whence $|V(s)-v| \leq C T=C_{T}$, thus $|v|^{p} \leq C\left(1+|V(s)|^{p}\right)$. Using this estimate and the fact that $Z(s)$ is a volume-preserving diffeomorphism yields the claim.

\section{References}

[1] Adams R.A., Sobolev Spaces, Academic Press, New York 1975

[2] Andréasson H., Global existence of smooth solutions in three dimensions for the semiconductor Vlasov-Poisson-Boltzmann equation, Nonlinear Anal. 28, 1193-1211 (1997)

[3] Bardos C.\& Degond P., Global existence for the Vlasov-Poisson equation in 3 space variables with small initial data, Ann. Inst. H. Poincaré Anal. Non Linéaire 2, 101-118 (1985)

[4] Batt J., Global symmetric solutions of the initial value problem in stellar dynamics, J. Differential Equations 25, 342-364 (1977)

[5] Blanchet L., Damour T.\& Schäfer G., Post-Newtonian hydrodynamics and post-Newtonian gravitational wave generation for numerical relativity, Mon. Not. R. Astron. Soc. 242, 289-305 (1990)

[6] Bouchut F., Existence and uniqueness of a global smooth solution for the Vlasov-Poisson-Fokker-Planck system in three dimensions, J. Funct. Anal. 111, 239-258 (1993) 
[7] Burke W.L., Gravitational radiation damping of slowly moving systems calculated using matched asymptotic expansions, J. Math. Phys. 12, 401-418 (1971)

[8] Flanagan É.É., Astrophysical sources of gravitational radiation and prospects for their detection, in Dadhich N. \& Narlikar J. (Eds.): Gravitation and Relativity at the Turn of the Millenium, Inter-University Centre for Astronomy and Astrophysics, Pune 1998

[9] Glassey R.T., The Cauchy Problem in Kinetic Theory, SIAM, Philadelphia 1996

[10] Hörmander L., The Analysis of Linear Partial Differential Operators I, Springer, Berlin-New York 1983

[11] Horst E., On the asymptotic growth of the solutions of the Vlasov-Poisson system, Math. Meth. Appl. Sci. 16, 75-85 (1993)

[12] Illner R. \& Rein G., Time decay of the solutions of the Vlasov-Poisson system in the plasma physical case, Math. Meth. Appl. Sci. 19, 1409-1413 (1996)

[13] Jackson J.D., Classical Electrodynamics, Wiley, New York 1975

[14] Kunze M.\& Spohn H., Radiation reaction and center manifolds, SIAM J. Math. Anal. 32, 30-53 (2000)

[15] Kunze M.\& Spohn H., Adiabatic limit for the Maxwell-Lorentz equations, Annales H. Poincaré 1, 625-653 (2000)

[16] Kunze M.\& Spohn H., Slow motion of charges interacting through the Maxwell field, Comm. Math. Phys. 212, 437-467 (2000)

[17] Lions P.L.\& Perthame B., Propagation of moments and regularity for the 3-dimensional Vlasov-Poisson system, Invent. Math. 105, 415-430 (1991)

[18] Perthame B., Time decay, propagation of low moments and dispersive effects for kinetic equations, Comm. Partial Differential Equations 21, 659-686 (1996)

[19] Pfaffelmoser K., Global classical solutions of the Vlasov-Poisson system in three dimensions for general initial data, J. Differential Equations 95, 281$303(1992)$

[20] Pulvirenti M.\& Simeoni C., $L^{\infty}$-estimates for the Vlasov-Poisson-FokkerPlanck equation, Math. Meth. Appl. Sci. 23, 923-935 (2000)

[21] Reed M.\& Simon B., Methods of Modern Mathematical Physics II: Fourier Analysis, Self Adjointness, Academic Press, New York 1975 
[22] Rein G., Selfgravitating systems in Newtonian theory - the Vlasov-Poisson system, in Proc. Minisemester on Math. Aspects of Theories of Gravitation 1996, Banach Center Publications 41, part I, 179-194 (1997)

[23] Rein G.\& Rendall A., Global existence of classical solutions to the Vlasov-Poisson system in a three dimensional, cosmological setting, Arch. Rat. Mech. Anal. 126, 183-201 (1994)

[24] Schaeffer J., Global existence of smooth solutions to the Vlasov-Poisson system in three dimensions, Comm. Partial Differential Equations 16,1313-1335 (1991)

[25] Zheleznyakov V.V., Radiation in Astrophysical Plasmas, Kluwer, Dordrecht 1996 